\title{
Data scheme-based wireless channel modeling method: motivation, principle and performance
}

\author{
Xiaochuan Ma, Jianhua Zhang*, Yuxiang Zhang, Zhanyu Ma \\ Beijing University of Posts and Telecommunications, Beijing 100876, China \\ *Corresponding author, Email: Jhzhang@bupt.edu.cn
}

\begin{abstract}
In recent years, data mining and machine learning technologies have made great progress driven by enormous volumes of data. Meanwhile, the wireless-channel measurement data appears large in volume because of the large-scale antenna numbers, increased bandwidth, and versatile application scenarios. With powerful data mining and machine learning methods and large volumes of data, we can extract valuable and hidden rules from the wireless channel. Motivated by this, we propose a channel-modeling method using PCA in this paper. Its principle is to utilize the features and structures extracted from the CIR data collected by measurements, and then model the wireless channel of the targeted measurement scenario. In addition, a noise removing method using a BP neural network is designed for the proposed model, which can recognize and remove the noise of the polluted CIR accurately. The performance of the proposed scheme is investigated with the actual measured CIR data, and its superiority is verified.
\end{abstract}

Keywords: MIMO, channel model, data mining, PCA, machine learning, neural network

\section{Introduction}

Nowadays, the ever-increasing numbers of users of smart phones, tablets, and other mobile devices and various applications have created enormous data traffic for wireless communication. Faced with the application scenario of an enhanced mobile internet, IMT-2020 (the International Mobile Telecom system-2020) or 5G (the 5th Generation) mobile communication system should provide access capability with higher data rates to support the explosive increase in multimedia contents characterized by higher definition and living experiences. To meet this demand, the spectral efficiency should be fur- ther improved, as there is a limitation on spectrum resources. MIMO (Multiple-Input and MultipleOutput) technique is an efficient way to improve the spectral efficiency, in that it can fully utilize the spatial resources to improve the channel capacity, and is widely used in wireless communication systems now. It will still be a mainstream technique in IMT-2020.

A wireless channel is the space that conveys information through electromagnetic waves between the transmitter antenna and receiver antenna. Its properties have a crucial influence on the performance of the wireless communication system. Hence, it is of great significance to study the MIMO channel for design, simulation, and deployment of wireless

Manuscript received Dec. 31, 2016; accepted Mar. 31, 2017

This work is supported by National Nature and Science Funding (Nos. 61322110, 61461136002), National Science and Technology Major Project (No. 2015ZX03002008-002), Ministry of Education-China Mobile Research Funding (No. 20160105), and Qualcomm Funding. 
communication systems. The mainstream modeling methods can be classified into two categories: the deterministic model and the GBSM (Geometry-Based Stochastic Model). The deterministic model is based on the electromagnetic field theory. The solutions or approximate solutions of Maxwell's equations are used to determine the CIR (Channel Impulse Response) of a location by detailed geographic information. It can generate the CIR very precisely when the propagation environment is completely built. On the other hand, as the deterministic model depends on the precision of the geographic information, its complexity is very high and its generalization ability is quite weak.

GBSMs are essentially stochastical channel models that empirically predict the fading of the received signal from the probability distribution and then reconstruct the CIR by using simulation methods in the sense of stochastics. The 3GPP SCM (Spatial Channel Model) was a classical GBSM of the B3G (Beyond 3rd Generation) wireless communication system $^{[1]}$. It supported frequencies and bandwidths up to $2 \mathrm{GHz}$ and $5 \mathrm{MHz}$, respectively. In the development of the $4 \mathrm{G}$ wireless communication, more channel models were created, such as WINNER I, WINNER II, and ITU-R M.2135 $5^{[2-4]}$. They could support higher frequencies, wider bandwidths, and more diverse scenarios than previous models. In recent years, with the emergence of 3D MIMO technology, the elevation dimension came to be considered ${ }^{[5-7]}$. In 2014, 3GPP released 3GPP TR 36.873 as the standard 3D channel model ${ }^{[8]}$.

The statistical characteristics of GBSMs, such as delay, amplitude, azimuth angle of arrival, azimuth angle of departure, elevation angle of departure, and Doppler shift are acquired from the measurement data. However, the correlations between the subchannels were scarcely embodied in traditional GBSMs. Channel capacity is an important metric to measure the effectiveness of a wireless channel. It is calculated from the FCIR (Frequency-Channel Impulse Response), and the correlations between subchannels have a significant influence on the channel capacity. Therefore, there are channel capacity de- viations between the traditional GBSM results and the measurement results. Especially, the deviation increases with an increase in the antenna element number. This will make the modeling of massive MIMO channels difficult, as they have large numbers of antennas.

To model the real channel precisely, new features and structures need to be extracted from the measurement data. Data mining is widely known as KDD (Knowledge Discovery in Databases ${ }^{[9]}$. It helps us to find hidden and potentially useful information from the repositories. Machine learning is a subfield of computer science. It also helps us to analyze and process the data. The difference between data mining and machine learning is that the former focuses more on exploratory data analysis and is known as unsupervised learning. They can be viewed as two facets of the same field ${ }^{[10]}$. Nevertheless, both of them are powerful tools, which help us to discover the rules hidden in the data and process the data efficiently. The methods of data mining and machine learning have been used in channel modeling. For example, the $K$-means algorithm was used to cluster the rays in Ref. [11], and the EM (ExpectationMaximization) algorithm has also been used to estimate the features of the multipaths in Ref. [12]. Recently, deep learning has been used in channel estimation and works well ${ }^{[13,14]}$.

PCA (Principal Component Analysis) is a statistical procedure that uses an orthogonal transformation to convert a set of observations of possibly correlated variables into a set of values of linearly uncorrelated variables called principal components ${ }^{[15]}$. PCA can find the principal information or features of the signal. PCA was used to redescribe the relationships between large-scale parameters of wireless channels in Ref. [16]. In this study, we apply PCA to find the principal features of FCIR. The BP neural network is very powerful in solving regression and classification tasks ${ }^{[17]}$. Here, a BP network is used to denoise the CIR acquired from the measurements.

In this paper, a channel modeling method is proposed to improve the accuracy of channel capacity without noticeable loss in accuracy of other chan- 
nel metrics. The contributions of this paper are as follows:

1) The BP neural network is exploited to recognize the efficient signal from the CIR and remove the noise.

2) The principal components of the FCIR's amplitude are acquired from the measurement data by PCA.

3) The features and structures of the FCIR are used to model the wireless channel.

The rest of this paper is organized as follows. In section 2, we describe our research motivation. In section 3 , we attempt to use two machine learning and data mining methods for wireless channel modeling. The MIMO measurement we carried out is described in section 4 . In section 5 , the modeling procedure is demonstrated. Finally, the paper is concluded in section 6 .

\section{Motivation}

Formerly, the channel measurement data of a singleinput-single-output communication system with 20 $\mathrm{MHz}$ bandwidth was less than $1 \mathrm{MB}$. With the increase in the antenna number and bandwidth in the wireless communication, the data size of a $32 \times 56$ MIMO channel measurement with a bandwidth of $200 \mathrm{MHz}$ has gone up to $1 \mathrm{~GB}^{[16]}$. In IMT-2020, the channel bandwidth will be as wide as $1 \mathrm{GHz}$. The antenna number in massive MIMO systems will also be further increased, so that the channel data is completely in conformity with the volume property of "Big data". The wireless channel prosperities vary with the different frequencies, bandwidths, and scenarios. Hence, it is rather important to figure out the rules of the variations from the channel data. The volume and variation properties of the channel data encourage us to apply data mining and machine learning methods to analyze the channel data, extract interesting channel properties and rules, and use them to model the channel precisely.

In MIMO channels, the time-variant response channel with $N_{\mathrm{Tx}}$ transmitting antennas and $N_{\mathrm{Rx}}$ receiving antennas is given by a complex matrix $\boldsymbol{H}$ of dimension $N_{\mathrm{Tx}} \times N_{\mathrm{Rx}}$, where $N_{\mathrm{Tx}}$ and $N_{\mathrm{Rx}}$ are the numbers of Tx and $\mathrm{Rx}$ antenna elements, respectively. Each resolvable path has its delay $\tau$. The channel complex matrix $\boldsymbol{H}(t, \tau)$ of delay $\tau$ at time $t$ is

$$
\boldsymbol{H}(t, \tau)=\sum_{i=1}^{N} \boldsymbol{H}_{i}(t, \tau)=\sum_{i=1}^{N} \boldsymbol{H}_{i}(t) \cdot \delta\left(\tau-\tau_{i}\right),
$$

where $\tau_{i}$ is the delay of the $n$th path, and $\delta(\cdot)$ is the Dirac delta function.

As previously stated, channel capacity is an important metric for the MIMO channel. For the CIR of every measurement location, the discrete Fourier transform is applied to obtain the $N_{\mathrm{Tx}} \times N_{\mathrm{Rx}}$ FCIR matrix, $\boldsymbol{H}(t, f)$, where $f$ denotes the subcarrier indices. The mean capacity of the measurement scenario is calculated by averaging the capacity of all the sub-carriers and all the measurement locations $^{[18]}$.

$$
\begin{aligned}
C= & \frac{1}{L N_{f}} \sum_{p=1}^{L} \sum_{f=1}^{N_{f}} \mathrm{lb} \operatorname{det}\left(\boldsymbol{I}_{N}\right. \\
& \left.+\frac{\rho}{\beta^{2}} N_{\mathrm{Tx}} \boldsymbol{H}(t, f) \boldsymbol{H}^{\mathrm{H}}(t, f)\right),
\end{aligned}
$$

where $\rho$ is the SNR (signal-to-noise ratio), $L$ and $N_{f}$ are the numbers of measurement locations and sub-carriers, respectively, and $N$ is the smallest one of $N_{\mathrm{Tx}}$ and $N_{\mathrm{Rx}}$. $\beta$ is a normalization factor to remove the effect of different path losses at different measurement locations, defined by

$$
\mathrm{E}\left[\frac{1}{\beta}\|\boldsymbol{H}(t, f)\|_{F}^{2}\right]=N_{\mathrm{Rx}} N_{\mathrm{Tx}} .
$$

Obviously, FCIR is decisive in the calculation of channel capacity. Therefore, without reasonably modeling the amplitude and phase of FCIR, it is impossible to get an accurate channel capacity by channel modeling. To model the FCIR, its features and structures should be elucidated. The FCIR at one measurement location is an $N_{s} \times N_{f}$ complex matrix, $N_{s}$ is the number of sub-channels; it is the product of $N_{\mathrm{Tx}}$ and $N_{\mathrm{Rx}}$. As the measurements were performed at $L$ different locations, an $N_{L S} \times N_{f}$ FCIR data set is obtained by combining the FCIRs of $L$ 
measurement locations, where $N_{L S}$ is the product of $L$ and $N_{s}$.

It is helpful that we have a large amount of channel data now, which will give us information about channels that is more accurate. However, the feature number of the large amount of data is also very large, so that it will make the data analysis fairly time consuming or even inaccessible. Therefore, the dimension of the data should be reduced. Data reduction technologies can be used to find a much smaller representation of the original data, and the vast majority of the original data remains in the new representation. Specifically, the new representation can be more efficiently analyzed without a noticeable loss of the original information. There are many methods for data reduction, such as RBM (Restricted Boltzmann Machine) and PCA. RBM is a very useful algorithm for dimensionality reduction, classification, regression, collaborative filtering, feature learning, and topic modeling ${ }^{[19]}$. It was invented by Hinton, and is a very important part of deep learning ${ }^{[20]}$. However, RBM is relatively complex. Hence, in this study we apply a much easier method, PCA. PCA can reduce the dimension of the FCIR data and find the principal components of the data.

In real wireless channels, except the multipath signal, there are noise signals in the CIR. The noise can lead to weaker correlations between the sub-channels and a higher channel capacity. Therefore, to analyze the multi-path signals accurately, the noise should be eliminated first. The traditional method to remove the noise is quite inflexible. In this paper, we use a neural network-based method to remove the noise smartly and accurately.

\section{Machine learning and data min- ing based wireless channel model- ing methods}

\subsection{Denoise the CIR using BP neural network}

The traditional method to denoise the CIR is to set a noise threshold, which is shown in Fig. 1. When the signal in one chip has a power higher than the threshold, it will be judged as an efficient signal, and noise otherwise. In the figure, it can be seen that when the threshold is set as the maximum power of the noise, as the green line, all noise will be eliminated, while a part of the efficient signal will be removed. On the contrary, when the threshold is set as the lowest power of the efficient signal, as the yellow line, the efficient signal will not be affected, but a part of noise will remain. Therefore, it is very difficult to remove the noise completely without affecting the efficient signals, using the traditional method.

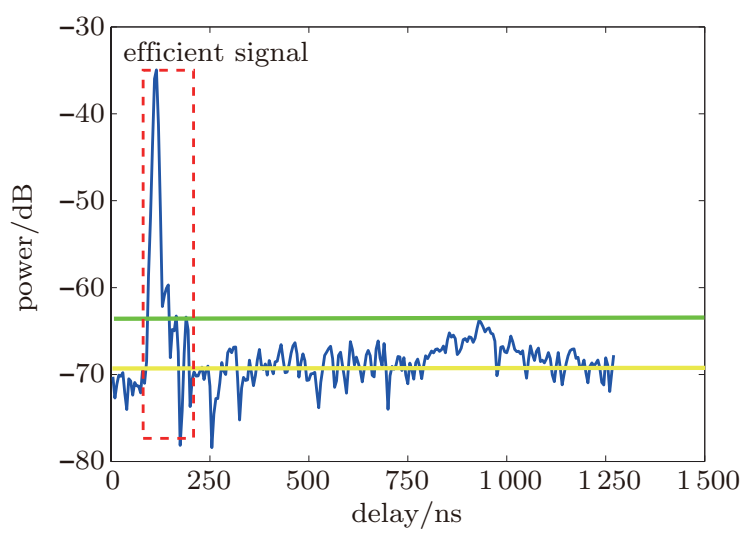

Figure 1 Traditional noise-removal method

Actually, efficient signal and noise can be abstracted into two classes. Thus, the denoising task becomes a common binary classification task. In machine learning, there are many algorithms which can solve this task successfully, such as neural networks and support vector machines ${ }^{[17,21]}$. Here, we choose neural networks because they are easy to implement and give good results.

Firstly, the data should be preprocessed as

$$
I_{i}=\frac{\boldsymbol{D}_{i}-\min (\boldsymbol{D})}{\max (\boldsymbol{D})-\min (\boldsymbol{D})}, \quad i=1,2,3, \cdots, 254,
$$

where $\boldsymbol{D}_{i}$ is the power value of the $i$ th chip of a PDP (power delay profile). In our data, the chip number is 254 (period of every chip is $5 \mathrm{~ns}$ ), so $\boldsymbol{D}$ is a vector consisting of all 254 power values in the PDP.

As shown in Fig. 2, we take the power value to be classified $\left(\boldsymbol{I}_{i}, i=1,2, \cdots, 254\right)$ and its six neighboring power values (three before the power value 
to be classified and three behind it $\left(\boldsymbol{I}_{i-n}, n=\right.$ $-3,-2,-1,1,2,3)$ as an input for the neural network. The label corresponding to the input$\boldsymbol{O}_{i}$ is the output of the neural network; the value of $\boldsymbol{O}_{i}$ is 1 (efficient signal) or 0 (noise). Thus far, at the $i$ th chip of the PDP, we have $\left\{\boldsymbol{I}_{i-3}, \boldsymbol{I}_{i-2}, \boldsymbol{I}_{i-1}, \boldsymbol{I}_{i}, \boldsymbol{I}_{i+1}, \boldsymbol{I}_{i+2}, \boldsymbol{I}_{i+3}, \boldsymbol{O}_{i}\right\}$; the eight values are defined as a sample.

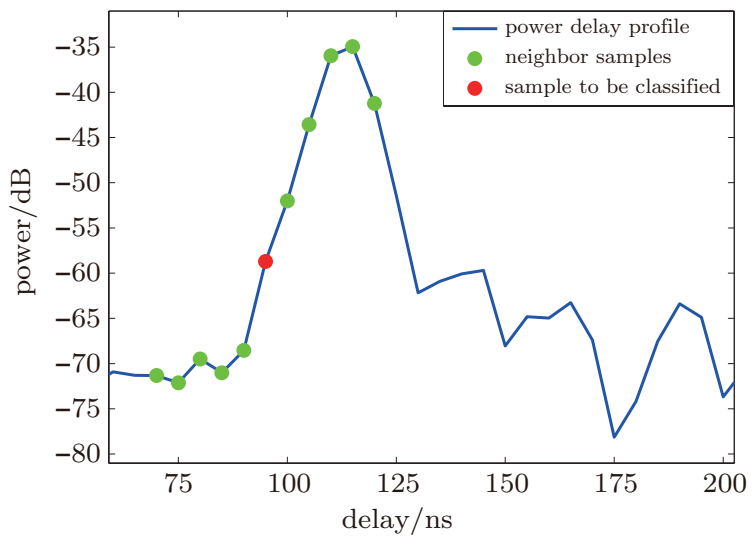

Figure 2 Input structure of the neural network

The neural network that we use has one input layer, two hidden layers (both have 40 nodes), and one output layer, the active function of every layer is a sigmoid function. The structure of the neural network is shown in Fig. 3.

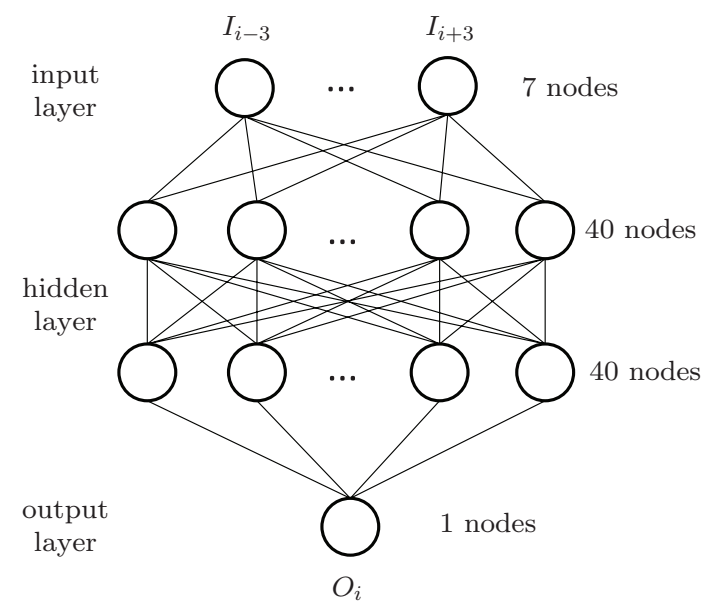

Figure 3 Structure of the BP neural network

There are 254 chips in one cycle. We manually classify 150 cycles of PDPs collected at different mea- surement locations. A hundred of these cycles are taken as training sets (25 400 samples) of the neural network, and the other 50 cycles as test sets (12800 samples). After training, the classification accuracy rate of the neural network for test sets reaches $95 \%$.

After the classification, the outputs of the 254 inputs form a $1 \times 254$ vector, which is denominated as an ESM (Efficient Signal Mask), as shown in Fig. 4. Multiplied by the ESM, the noise in CIR will be removed precisely.

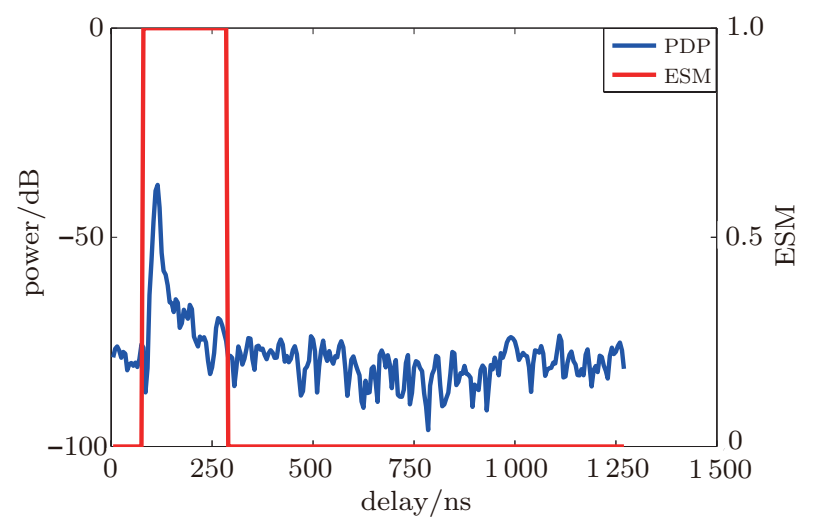

Figure 4 Noise removal using neural networks

\subsection{Analysis of the FCIR}

As the FCIR data set is a complex matrix, the AFCIR (Amplitude of the FCIR) and the PFCIR (phase of the FCIR) are analyzed.

Firstly, we analyze the AFCIR. The AFCIRs of the $L$ measurement locations are combined to an $N_{L S} \times N_{f}$ AFCIR matrix $\boldsymbol{A}$. PCA was then used to analyze the AFCIR matrix. The procedure of AFCIR analysis is shown in Fig. 5. It should be noted that the AFCIRs are all normalized in every subchannel, and the power of every sub-channel can be precisely calculated by using a traditional 3D channel model such as TR 36.873 .

1) Calculate mean values of every row of $\boldsymbol{A}$ and place them into an $N_{L S} \times 1$ vector $\boldsymbol{u}$.

$$
\boldsymbol{u}(m)=\frac{1}{N_{f}} \sum_{n=1}^{N_{f}} \boldsymbol{A}(m, n), \quad m=1,2, \cdots, N_{L S} .
$$

2) Centralize $\boldsymbol{A}$ as

$$
\mathbf{S}=\boldsymbol{A}-\boldsymbol{h} \boldsymbol{u}^{\mathrm{T}} .
$$


where $\boldsymbol{h}$ is a $N_{f} \times 1$ column vector of all $1 \mathrm{~s}$.

3) The autocorrelation matrix of $\boldsymbol{S}$ is derived as $\boldsymbol{C}=\boldsymbol{S}^{\mathrm{T}} \boldsymbol{S}$, and then an eigenvalue decomposition is applied on $\boldsymbol{C}$.

$$
\mathbf{C}=\boldsymbol{F} \boldsymbol{\Sigma} \boldsymbol{F}^{-1},
$$

where $\boldsymbol{F}=\left[\boldsymbol{f}_{1}, \cdots, \boldsymbol{f}_{N_{f}}\right]$ and $\boldsymbol{\Sigma}=\operatorname{diag}\left[\lambda_{1}, \cdots, \lambda_{N_{f}}\right]$, $\boldsymbol{f}_{i}$ and $\lambda_{i}$ are the eigenvectors and eigenvalues, respectively.

4) Normalize the eigenvalues in eigenvector $\boldsymbol{\Sigma}$.

$$
\lambda_{i}=\frac{\lambda_{i}}{\sum_{n=1}^{N_{f}} \lambda_{n}}, \quad i=1, \cdots, N_{f} .
$$

5) Calculate the projection matrix $\boldsymbol{T}$ as

$$
T=S F
$$

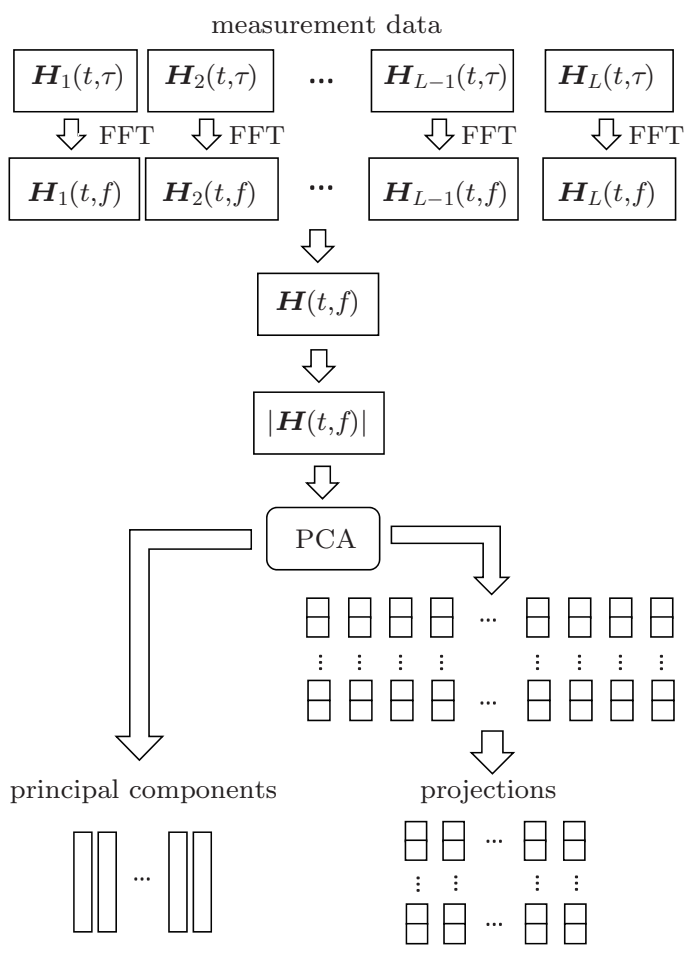

Figure 5 Analysis of the FCIR

So far, the PCA procedure on the AFCIR in $N_{L S}$ sub-channels is completed, and three results, viz., $\boldsymbol{F}$, $\boldsymbol{T}$, and $\boldsymbol{\Sigma}$ are acquired. Every column of $\boldsymbol{F}$ is a component vector of $\boldsymbol{S}$. $\boldsymbol{T}$ has $N_{L S}$ rows, and each of them is a projection vector on the $N_{f}$ components of a row of $\boldsymbol{S}$. In the present study, if the sum of the largest $p$ eigenvalues in $\boldsymbol{\Sigma}$ is more than $90 \%$, the corresponding $p$ columns in $\boldsymbol{F}$ are chosen as the principal components of AFCIRs in the scenario. The $p$ values corresponding to the principal components in every column of $\boldsymbol{T}$ are the $p$ projections of the AFCIR of every sub-channel on the $p$ principal component vectors. With the principal components and the corresponding projections, the original AFCIRs can be reconstructed. The correlation coefficients between the $p$ projections in $N_{L S}$ sub-channels are very low ; the highest correlation coefficients between them is 0.3 in our data analysis. Therefore, the $p$ projections are assumed to be independent. The distribution of the $p$ projection in the $N_{L S}$ sub-channels can be acquired from matrix $\boldsymbol{T}$. After the principal components and projection vectors are acquired, the normalized AFCIR matrix for one measurement location in the measurement scenario can be acquired. With the help of a traditional GBSM such as TR 36.873 , the power values of all sub-channels at different measurement locations are acquired. Finally, the AFCIR of different Rx and Tx locations can be generated.

After the AFCIR is generated, the PFCIR can be acquired according to the AFCIR and statistical properties. As the AFCIR is generated, the PFCIR can determine the properties of the time domain and correlations between the sub-channels. According to the IDFT (Inverse Discrete Fourier Transform) formula, the phase in every sub-channel can determine the PDP of the sub-channel. Hence, we can reasonably set the PFCIR in every sub-channel to obtain the time-domain properties. On the other hand, the phase deviations between every corresponding element of the PFCIR vectors corresponding to two Tx antenna elements can determine the correlations between the sub-channels. If the phase deviations can be set reasonably, the correlations between the subchannels can be precisely modeled. In this study, we set the phase deviations empirically according to the relative locations and polarizations of the antenna elements. The PFCIR can then be generated. Finally, the FCIR can be acquired. 


\section{Measurement description}

To provide data for PCA and validate the proposed modeling method, an indoor measurement is performed.

\subsection{Measurement system}

To capture real channel information, we utilized the Elektrobit Propsound sounder described in Ref. [22] which worked in time-division multiplexing mode by transmitting periodic pseudorandom binary sequence (m-sequence). The transmitter modulated an m-sequence at a carrier frequency of $6 \mathrm{GHz}$, and the signal bandwidth was $100 \mathrm{MHz}$. We defined the interval such that all antenna elements were sounded once as a measurement cycle (snapshot). Every cycle had 254 chips, 1270 ns. The ASU (AntennaSwitching Units) were connected to Tx and Rx antenna arrays to control the signal transmitted from Tx and the signal received at Rx, respectively. To capture the rays in a $3 \mathrm{D}$ environment, the system was equipped with $3 \mathrm{D}$ antenna arrays. As shown in Fig. 6, a dual-polarized ODA (Omnidirectional Array) consisting of 56 antenna elements with eight adjacent sides and a top surface was used at Rx, and another identical ODA, which acted only 32 of the 56 elements, was used at Tx. The specifications of our measurement are shown in Tab. 1. The Tx and Rx ODAs were calibrated in an anechoic chamber.

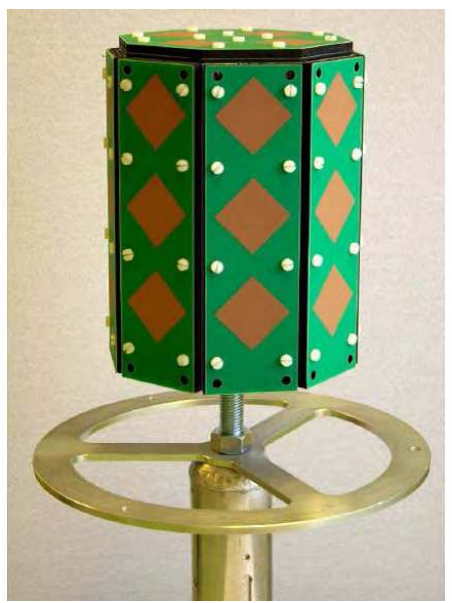

Figure 6 ODA with 56 antenna elements
Table 1 The specifications of measurement

\begin{tabular}{ccc}
\hline parameter & \multicolumn{3}{c}{ value } \\
\hline antenna type & ODA(Rx) & ODA(Tx $)$ \\
$\begin{array}{c}\text { number of } \\
\text { antenna ports } \\
\text { number of } \\
\text { elements } \\
\text { angle azimuth }\end{array}$ & 56 dual polarized & 28 dual polarized \\
range elevation & $\left(-180^{\circ}, \quad 180^{\circ}\right)$ & $\left(-180^{\circ}, \quad 180^{\circ}\right)$ \\
polarization & $\left(-70^{\circ}, \quad 90^{\circ}\right)$ & $\left(-70^{\circ}, \quad 90^{\circ}\right)$ \\
center frequency & $\pm 45^{\circ}$ & $\pm 45^{\circ}$ \\
bandwidth & \multicolumn{3}{c}{$6 \mathrm{GHz}$} \\
PN sequence & \multicolumn{3}{c}{$100 \mathrm{MHz}$} \\
\hline
\end{tabular}

\subsection{Measurement scenario}

The measurement was performed in a teaching building of BUPT, China. The layout of the measurement is illustrated in Fig. 7. In our measurement, the BS (Base Station) with a $2.1 \mathrm{~m}$ high Tx antenna was installed at one end of a $15 \mathrm{~m}$ long corridor. The MS (Mobile Station) equipped with a $1.7 \mathrm{~m}$ high Rx antenna was located on a hand trolley. The Tx antenna pointed west while the Rx antenna pointed east. The Rx was measured at 179 different positions; the distance between the neighboring positions was $0.4 \mathrm{~m}$. Two hundred cycles were recorded at each $\mathrm{Rx}$ location. All the 179 positions were in the same corridor with the Tx, so that they had the LoS (Line of Sight) condition. The walls along the corridor were

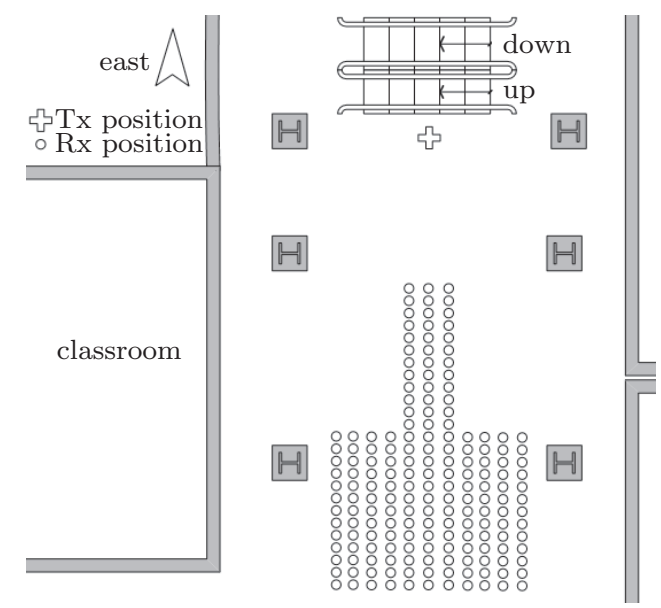

Figure 7 Layout of the measurement 
made of bricks with plasterboard on the surface. The floor was covered with marble, while the doors of the rooms were wooden. For a static channel condition, the measurement was performed when there was nobody between the Tx and $\mathrm{Rx}$.

\section{Channel modeling with FCIR fea- tures}

After the measurement, the FCIRs collected at 179 locations were combined to form an FCIR matrix. Following the method described in section 2, the wireless channel of the measured scenario was analyzed and modeled as follows. The modeling procedure is shown in Fig. 8.

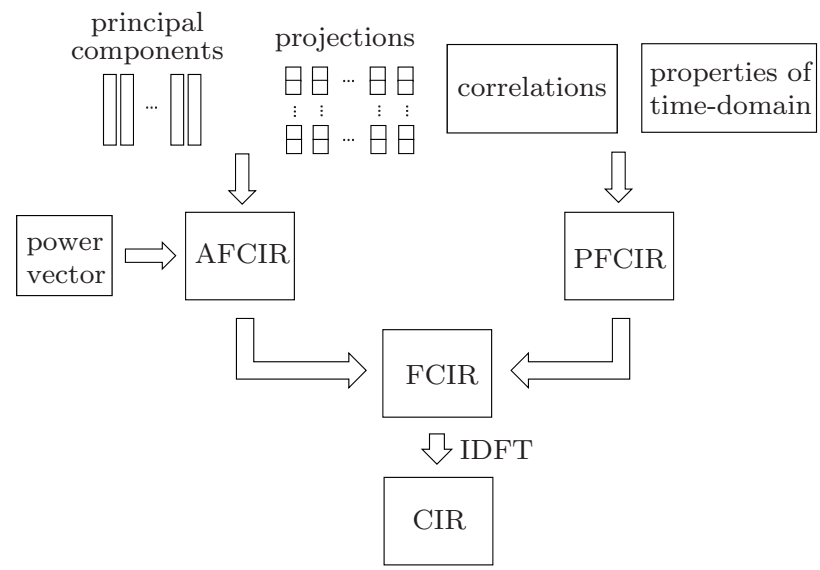

Figure 8 Modeling procedure of the FCIR

Given the Tx and Rx locations, an FCIR matrix CIR is generated according to the following steps.

1) The measurement data should be denoised by the neural network. The resulting PDP is shown in Fig. 9.

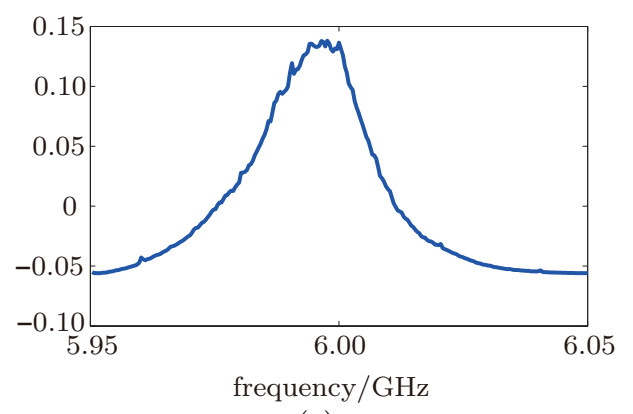

(a)

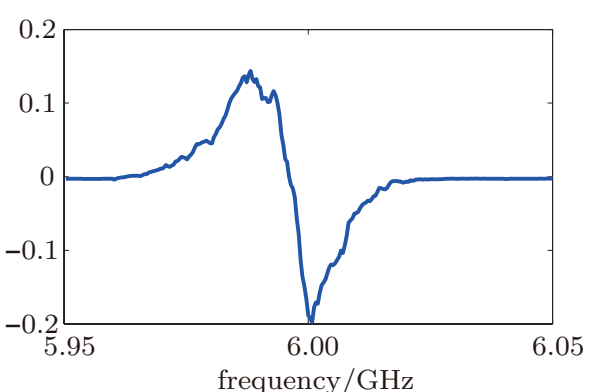

(b)

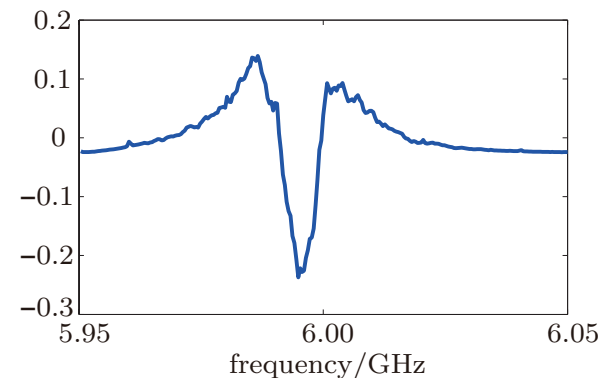

(c)

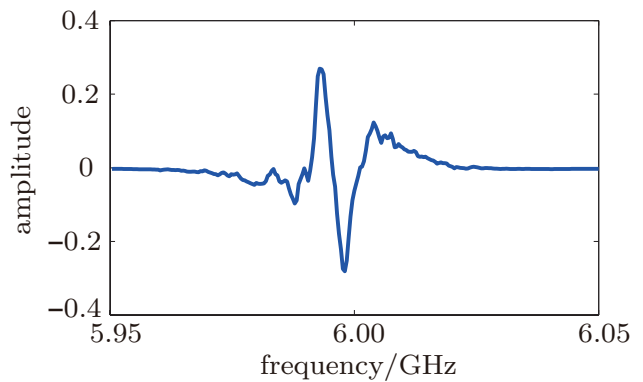

(d)

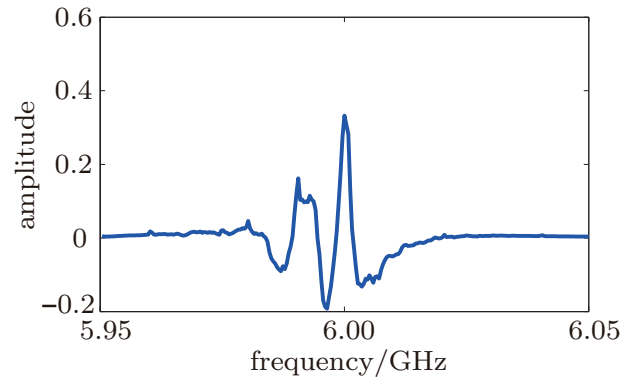

(e)

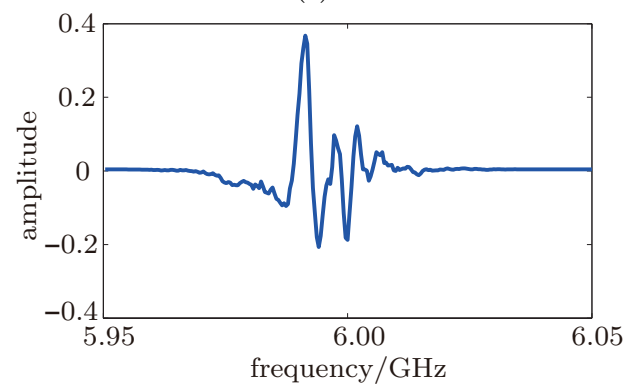

(f)

Figure 9 Principal components of AFCIR: (a) feature 1; (b) feature 2 ; (c) feature 3 ; (d) feature 4 ; (e) feature 5 ; (f) feature 6 
2) PCA is applied to the normalized AFCIR matrix of the measurement and three matrices $\boldsymbol{F}, \boldsymbol{T}$, and $\boldsymbol{\Sigma}$ are acquired. As the sum of the six largest eigenvalues in $\boldsymbol{\Sigma}$ is $91 \%$, the six corresponding components in matrix $\boldsymbol{F}$ are principal components of AFCIRs in these scenarios, and placed in a matrix $\boldsymbol{F}_{r}$. The six principal components are shown in Fig. 10. In addition, the distributions of six projections are extracted from matrix $\boldsymbol{T}$.

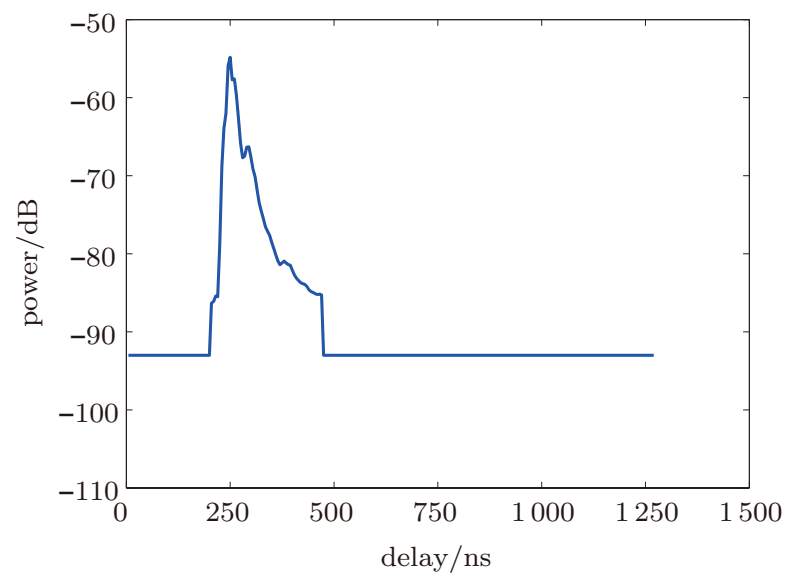

Figure 10 PDP after the noise removing

3) Calculate the normalization of $\boldsymbol{A}^{\prime}, \boldsymbol{A}_{N}$. A $1792 \times 6$ projection matrix $\boldsymbol{T}_{r}$ is generated randomly according to the distributions of the six projections. With the projections and the principal components, the $\boldsymbol{A}_{N}$ can be acquired. Finally, $\boldsymbol{A}_{N}$ should be normalized in every row to make every sub-channel in $\boldsymbol{A}_{N}$ has unit power.

4) Calculate $\boldsymbol{A}^{\prime}$. According to the $\mathrm{Tx}$ and $\mathrm{Rx}$ locations, the power values of all sub-channels are acquired from the TR 36.873 simulation under our measurement scenario and configuration, and then the square root values of the power values are placed in a $1792 \times 1$ vector $\boldsymbol{e}=\left\{e_{1}, e_{2}, \cdots, e_{1792}\right\}$. Finally, $\boldsymbol{A}^{\prime}$ is calculated as

$$
\boldsymbol{A}_{i}^{\prime}=e_{i} \boldsymbol{A}_{N i}, \quad i=1,2, \cdots, 1792,
$$

where $\boldsymbol{A}_{i}^{\prime}$ and $\boldsymbol{A}_{N i}$ are the $i$ th rows of $\boldsymbol{A}^{\prime}$ and $\boldsymbol{A}_{N}$, respectively.

5) The PFCIR matrix is generated according to the time-domain properties and channel capacities.
6) The FCIR matrix $\boldsymbol{H}_{f}^{\prime}$ is calculated as

$$
\begin{aligned}
& \boldsymbol{H}_{f}^{\prime}(m, n)=\boldsymbol{A}^{\prime}(m, n) \mathrm{e}^{-\mathrm{j} * \boldsymbol{P}^{\prime}(m, n)}, \\
& m=1,2, \cdots, 1792, \\
& n=1,2, \cdots, 254 .
\end{aligned}
$$

7) $\boldsymbol{H}_{f}^{\prime}$ is then transformed to a CIR matrix $\boldsymbol{H}$ by IDFT. Finally, $\boldsymbol{H}$ should be denoised by the neural network. At this stage, the whole modeling procedure is accomplished. The generated PDP is shown in Fig. 11. The channel capacities from the measured data, TR 36.873 , and the proposed model are shown in Fig. 12. It can be seen that the channel estimation of our model is more accurate than that of TR 36.873. Actually, with an SNR of $30 \mathrm{~dB}$, the accuracy of $56 \times 32$ MIMO capacity improves $12.8 \%$ when compared with TR 36.873 .

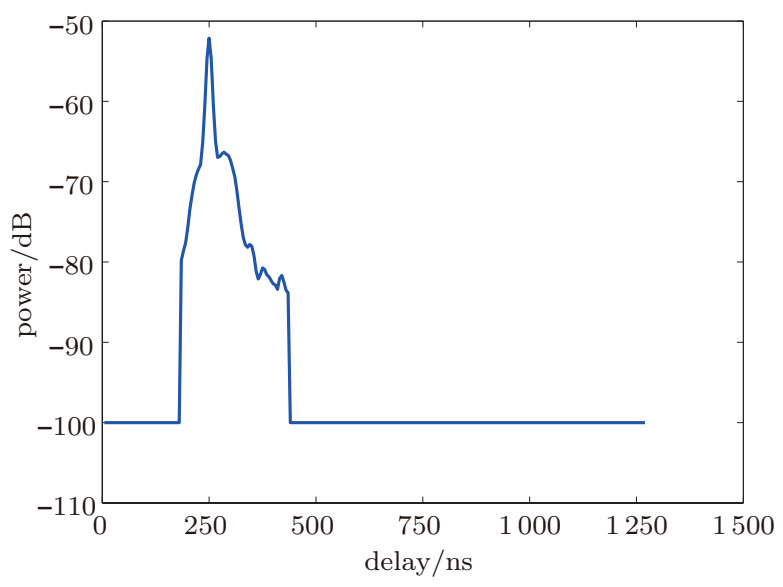

Figure 11 PDP of the proposed model

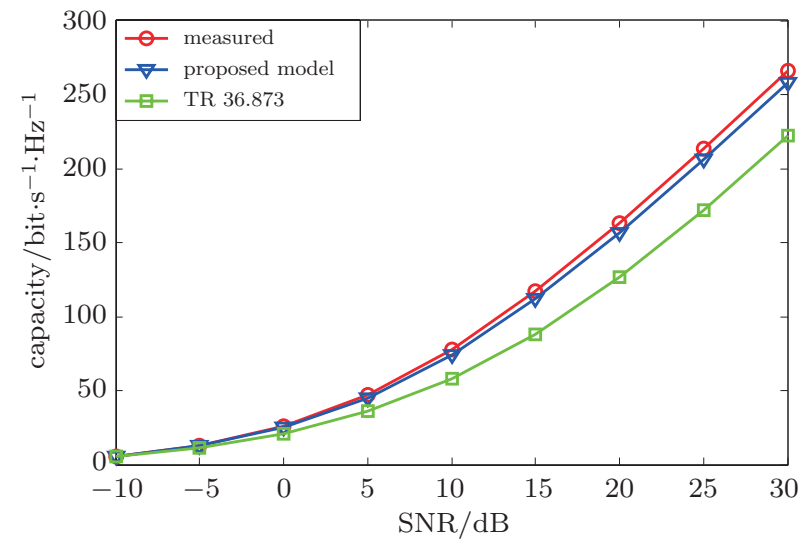

Figure 12 Channel capacities from the measured data, TR 36.873 , and the proposed model 


\section{Conclusion}

As the channel data has the prosperity of big data, this study attempts two machine learning and data mining methods in channel modeling. Firstly, as the traditional noise-removal method is inflexible, we proposed a neural network based noise-removal method. We trained the neural network with a training set which was manually acquired from the measurement data. Then load testing is set to the neural network. Consequently, the neural network can recognize and remove the noise smartly and precisely. Secondly, as the correlations of the sub-channels are scarcely embodied in the traditional GBSMs, their channel capacities are not accurate. As the FCIR is relatively important in the calculation of channel capacity, we study the features of FCIR. We use the PCA to extract the principal components of the FCIR in the measurement scenario. With the projection distributions corresponding to the principal components, we can reconstruct the AFCIR statistically. The PFCIR is set according to the correlations between the sub-channels and the time-domain properties of CIRs in this scenario. Finally, we can obtain the CIRs of different measurement locations in this scenario. With an SNR of $30 \mathrm{~dB}$, the prediction of $56 \times 32$ MIMO capacity is more accurate than that of TR 36.873 .

\section{References}

[1] 3GPP TSG RAN. Spatial channel model for multiple input multiple output (MIMO) simulations [S]. UK, Tech. 2004, 25(12.0).

[2] WINNER. Final report on link level and system level channel models [R]. WINNER, 2005.

[3] WINNER. IST-4-027756 WINNER II D1. 1.2 V1. 2 WINNER II Channel Models [R]. WINNER, 2007.

[4] International Telecommunication Union. Guidelines for evaluation of radio interface technologies for IMTAdvanced[R]. ITU, 2009.

[5] J. H. Zhang, C. Pan, F. Pei, et al. Three-dimensional fading channel models: A survey of elevation angle research [J]. IEEE communications magazine, 2014, 52(6): 218-226.

[6] X. Cheng, B. Yu, L. Yang, et al. Communicating in the real world: 3D MIMO [J]. IEEE wireless communica- tions, 2014, 21(4): 136-144.

[7] F. Pei, J. H. Zhang, C. Pan. Elevation angle characteristics of urban wireless propagation environment at 3.5 GHz [C]//IEEE 78th Vehicular Technology Conference (VTC Fall), 2013: 1-5.

[8] 3rd Generation Partenership Project. Technical Specification Group Radio Access Network; Study on 3D channel model for LTE (Release 12) [R]. UK: 3GPP. 2014.

[9] N. Padhy, D. Mishra, R. Panigrahi. The survey of data mining applications and feature scope $[\mathrm{J}]$. arXiv preprint arXiv:1211.5723, 2012.

[10] J. H. Friedman. Data mining and statistics: What's the connection? [J]. Computing science and statistics, 1998, 29(1): 3-9.

[11] N. Czink, P. Cera, J. Salo, et al. A framework for automatic clustering of parametric MIMO channel data including path powers $[\mathrm{C}] / /$ Vehicular Technology Conference, 2006: 1-5.

[12] B. H. Fleury, P. Jourdan, Stucki A. High-resolution channel parameter estimation for MIMO applications using the SAGE algorithm $[\mathrm{C}] / /$ International Zurich Seminar on Broadband Communications AccessTransmission-Networking, 2002: 30.

[13] F. N. Khan, C. Lu, A. P. T. Lau. Joint modulation format/bit-rate classification and signal-to-noise ratio estimation in multipath fading channels using deep machine learning [J]. Electronics letters, 2016, 52(14): $1272-1274$.

[14] X. Wang, L. Gao, S. Mao. PhaseFi: Phase fingerprinting for indoor localization with a deep learning approach [C]//IEEE Global Communications Conference (GLOBECOM), 2015: 1-6.

[15] I. Jolliffe. Principal component analysis [M]. Wiley Online Labrary, Ltd., 2002.

[16] J. Zhang. The interdisciplinary research of big data and wireless channel: a cluster-nuclei based channel model [J]. China communications, 2016, 13(Supplement2): 14-26.

[17] S. E. Dreyfus. Artificial neural networks, back propagation, and the Kelley-Bryson gradient procedure [J]. Journal of guidance, control, and dynamics, 1990, 13(5): 926-928.

[18] A. F. Molisch, M. Steinbauer, M. Toeltsch, et al. Capacity of MIMO systems based on measured wireless channels [J]. IEEE journal on selected areas in communications, 2002, 20(3): 561-569.

[19] Y. W. Teh, G. E. Hinton. Rate-coded restricted Boltzmann machines for face recognition [J]. Advances in neural information processing systems, 2001: 908-914.

[20] G. E. Hinton, R. R. Salakhutdinov. Reducing the dimensionality of data with neural networks $[\mathrm{J}]$. Science, 2006, 313(5786): 504-507.

[21] C. Cortes, V. Vapnik. Support-vector networks [J]. Machine learning, 1995, 20(3): 273-297.

[22] J. H. Zhang. Review of wideband MIMO channel mea- 
surement and modeling for IMT-Advanced systems [J]. Chinese science bulletin, 2012, 57(19): 2387-2400.

\section{About the authors}

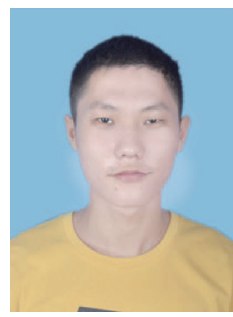

Xiaochuan Ma received the B.E. degree in electronic engineering from Communication university of China. He is now a M.S. candidate of Beijing University of Posts and Telecommunications. His research interests include channel modeling and data mining. (Email: xcma@bupt.edu.cn)

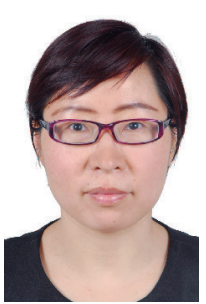

Jianhua Zhang [corresponding author] received the Ph.D. degree from Beijing University of Posts and Telecommunications. She is now a professor of BUPT. Her research interests include channel modeling and machine learning. (Email: Jhzhang@bupt.edu.cn)

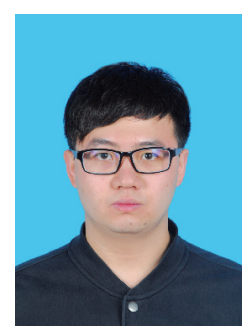

Yuxiang Zhang received the B.E. degree in electronic engineering from Dalian University of Technology. He is now a Ph.D. candidate of Beijing University of Posts and Telecommunications. His research interests include channel modeling and data mining. (Email: zhangyx@bupt.edu.cn)

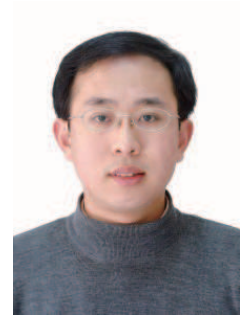

Zhanyu Ma received the Ph.D. degree from Kungliga Tekniska Hogskolan. He is now an associate professor of Beijing University of Posts and Telecommunications. His research interests include pattern recognition and machine learning. (Email: mazhanyu@bupt.edu.cn) 